\title{
Climate Variability Across Scales: from centuries to millennia (CVAS)
}

Shaun Lovejoy ${ }^{1}$ and CVAS Science committee ${ }^{2}$

"Weather is what you get, climate is what you expect". Is it, really? Consider a series of measurements of temperature along the time axis (Fig. 1A). If successive fluctuations tend to cancel each other (Fig. 1B), then averaging over longer and longer intervals smooths the signal; the averages converge more and more to an (apparently) well-defined mean value. However, if slow processes are forcing the system - for example changing the albedo or $\mathrm{CO}_{2}$ - the system changes constantly, so that this apparently convergent mean slowly varies, and choosing longer and longer periods to estimate it will be of no help. Such systems "wander": they do not "relax" (Fig. 1C). Whether the system is relaxing or wandering is a matter of timescales: at weather scales or at the multi-millennium (climate) scales, it wanders. Predicting the future thus requires knowledge of the entire history of the evolution of the system.

Therefore, we need to take a fresh look at climate variability and theory, bringing together nonlinear geoscientists and climate scientists to think about effective ways to (i) characterize climate fluctuations and understand their causes and (ii) focus on these aspects when analyzing both climate data and climate models.

This is the objective of the new PAGES working group on "Climate Variability Across Scales: from centuries to millennia (CVAS)". The group brings people together from

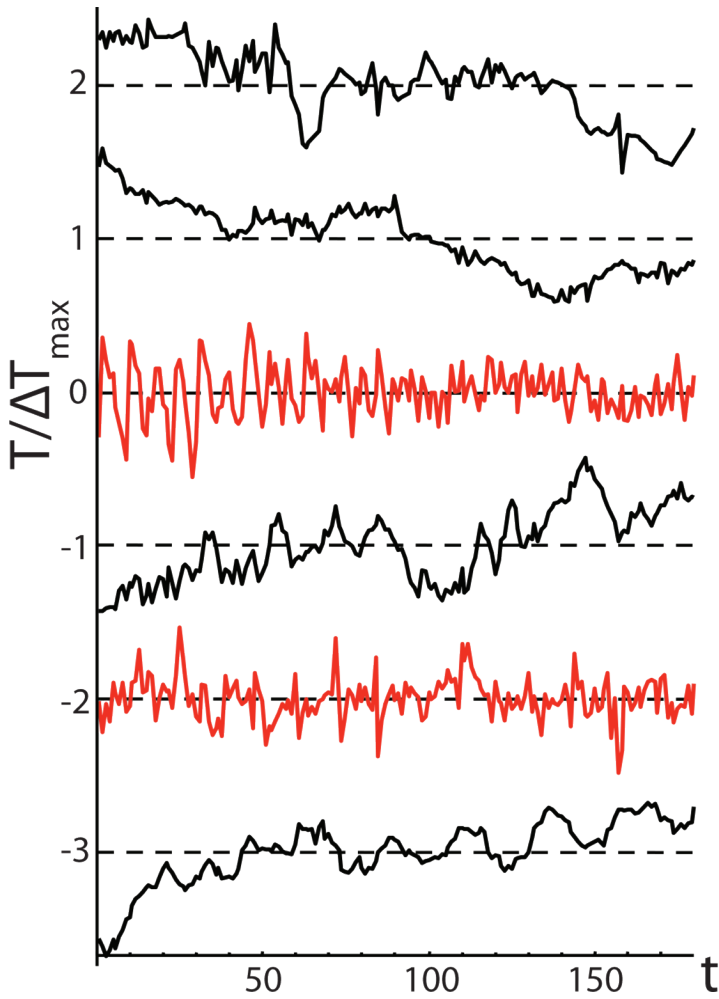

\section{F - Megaclimate} Veizer: 290-511 Myrs BP (1.23 Myr) $\mathrm{H} \approx 0.4$

\section{E - Megaclimate}

Zachos: 0-67 Myrs BP (370 kyr) $\mathrm{H} \approx 0.4$

\section{D - Macroclimate} Huybers: 0-2.56 Myrs BP (14 kyr) $\mathrm{H} \approx-0.8$

\section{C - Climate}

Epica: 25-97 kyrs BP (400 yr) $\mathrm{H} \approx 0.4$

\section{B - Macroweather}

Berkeley: 1880-1895 AD (1 month) $\mathrm{H} \approx-0.4$

\section{A - Weather}

Lander Wyoming July 2005 (1 hour) $\mathrm{H} \approx 0.4$

Figure 1: Representative series from each of the five scaling regimes over time periods of seconds to hundreds of millions of years, resolutions indicated in parenthesis with daily, annual detrending (when needed). Starting at the bottom, (A) the temperature seems to wander, the difference between two values tends to increase with lag: for the fluctuation $\Delta T$ over time interval (lag) $\Delta t$, analysis shows that $\Delta T \approx \Delta t^{H}$ with $H>0$. (B) On the contrary, the Berkeley (global, land) series has successive fluctuations that tend to cancel; $H<0$ quantifies the rate. Moving upward, (C) the Epica Antarctic $\delta^{18} \mathrm{O}$ based paleo series is again "wandering". (D-F) The top three are based on benthic isotopes. Each series had the same number of points (180) and was normalized by its overall range, and was offset by 1 unit in the vertical for clarity. The black curves all illustrate "wandering" cases with $H>0$, the red "canceling", with $H<0$. This figure is reproduced from Lovejoy (2015) where full details are given.

both climate and nonlinear geophysics, with expertise in climate, paleoclimate, climate modeling, nonlinear physics, statistics and stochastics. The group's common research agenda includes a number of themes around the variability of the climate, especially at centennial to multi-millennial scales.

Examples of research that the working group will highlight include: pitfalls while exploiting state-of-the-art paleoclimate data; the origin of long-memory processes; spatiotemporal variability; the gain between forcing and response at timescales of hundreds and thousand years; the carbon budget; atmosphere dynamics at the Last Glacial Maximum; and the role of long-memory processes for seasonal, annual and decadal forecasts, for extreme events and for global warming.

Specific activities include (i) the development of statistical and modeling tools for analyzing and comparing time series and spatial distributions, focusing on centennial and millennial timescales, (ii) the continued development of numerical packages and emphasis of training aspects, through maintained webpages and mini-courses, (iii) preparation of paleoclimate compilations (in both space and time), consistent with respect to their centennial and millennial scale variability, and to properly account for the role of variability in proxy recording on top of climate variability, and (iv) provision of open-source and easy to use software for above data analysis.

The group will hold its first workshop, titled "What do we know about multicentennial, multimillenial variability?"in Hamburg, Germany, from 28-30 November 2016.

\section{AFFILIATIONS}

'Department of Physics, McGill University, Montreal, Canada

${ }^{2}$ www.pastglobalchanges.org/ini/wg/cvas/people

CONTACT

Shaun Lovejoy: lovejoy@physics.mcgill.ca

LINK

www.pastglobalchanges.org/ini/wg/cvas/intro REFERENCES

Lovejoy S (2015) Climate Dyn 44: 3187-3210 